\title{
Existence and Uniqueness of Solutions for Higher-Order Three-Point Boundary Value Problems
}

\author{
Minghe Pei ${ }^{1}$ and Sung Kag Chang ${ }^{2}$ \\ ${ }^{1}$ Department of Mathematics, Bei Hua University, JiLin 132013, China \\ ${ }^{2}$ Department of Mathematics, Yeungnam University, Kyongsan 712-749, South Korea
}

Correspondence should be addressed to Sung Kag Chang, skchang@ynu.ac.kr

Received 5 February 2009; Accepted 14 July 2009

Recommended by Kanishka Perera

We are concerned with the higher-order nonlinear three-point boundary value problems: $x^{(n)}=$ $f\left(t, x, x^{\prime}, \ldots, x^{(n-1)}\right), n \geq 3$, with the three point boundary conditions $g\left(x(a), x^{\prime}(a), \ldots, x^{(n-1)}(a)\right)=$ $0 ; x^{(i)}(b)=\mu_{i}, i=0,1, \ldots, n-3 ; h\left(x(c), x^{\prime}(c), \ldots, x^{(n-1)}(c)\right)=0$, where $a<b<c, f:[a, c] \times \mathbb{R}^{n} \rightarrow$ $\mathbb{R}=(-\infty,+\infty)$ is continuous, $g, h: \mathbb{R}^{n} \rightarrow \mathbb{R}$ are continuous, and $\mu_{i} \in \mathbb{R}, i=0,1, \ldots, n-3$ are arbitrary given constants. The existence and uniqueness results are obtained by using the method of upper and lower solutions together with Leray-Schauder degree theory. We give two examples to demonstrate our result.

Copyright (C) 2009 M. Pei and S. K. Chang. This is an open access article distributed under the Creative Commons Attribution License, which permits unrestricted use, distribution, and reproduction in any medium, provided the original work is properly cited.

\section{Introduction}

Higher-order boundary value problems were discussed in many papers in recent years; for instance, see [1-22] and references therein. However, most of all the boundary conditions in the above-mentioned references are for two-point boundary conditions [2-11, 14, 17-22], and three-point boundary conditions are rarely seen $[1,12,13,16,18]$. Furthermore works for nonlinear three point boundary conditions are quite rare in literatures.

The purpose of this article is to study the existence and uniqueness of solutions for higher order nonlinear three point boundary value problem

$$
x^{(n)}=f\left(t, x, x^{\prime}, \ldots, x^{(n-1)}\right), \quad n \geq 3,
$$


with nonlinear three point boundary conditions

$$
\begin{gathered}
g\left(x(a), x^{\prime}(a), \ldots, x^{(n-1)}(a)\right)=0, \\
x^{(i)}(b)=\mu_{i}, \quad i=0,1, \ldots, n-3, \\
h\left(x(c), x^{\prime}(c), \ldots, x^{(n-1)}(c)\right)=0,
\end{gathered}
$$

where $a<b<c, f:[a, c] \times \mathbb{R}^{n} \rightarrow \mathbb{R}=(-\infty,+\infty)$ is a continuous function, $g, h: \mathbb{R}^{n} \rightarrow \mathbb{R}$ are continuous functions, and $\mu_{i} \in \mathbb{R}, i=0,1, \ldots, n-3$ are arbitrary given constants. The tools we mainly used are the method of upper and lower solutions and Leray-Schauder degree theory.

Note that for the cases of $a=b$ or $b=c$ in the boundary conditions (1.2), our theorems hold also true. However, for brevity we exclude such cases in this paper.

\section{Preliminary}

In this section, we present some definitions and lemmas that are needed to our main results.

Definition 2.1. $\alpha(t), \beta(t) \in C^{n}[a, c]$ are called lower and upper solutions of BVP (1.1), (1.2), respectively, if

$$
\begin{aligned}
\alpha^{(n)}(t) \geq & f\left(t, \alpha(t), \alpha^{\prime}(t), \ldots, \alpha^{(n-1)}(t)\right), \quad t \in[a, c], \\
& g\left(\alpha(a), \alpha^{\prime}(a), \ldots, \alpha^{(n-1)}(a)\right) \leq 0, \\
& \alpha^{(i)}(b) \leq \mu_{i}, \quad i=0,1, \ldots, n-3, \\
& h\left(\alpha(c), \alpha^{\prime}(c), \ldots, \alpha^{(n-1)}(c)\right) \leq 0, \\
\beta^{(n)}(t) \leq & f\left(t, \beta(t), \beta^{\prime}(t), \ldots, \beta^{(n-1)}(t)\right), \quad t \in[a, c], \\
& g\left(\beta(a), \beta^{\prime}(a), \ldots, \beta^{(n-1)}(a)\right) \geq 0, \\
& \beta^{(i)}(b) \geq \mu_{i}, \quad i=0,1, \ldots, n-3, \\
& h\left(\beta(c), \beta^{\prime}(c), \ldots, \beta^{(n-1)}(c)\right) \geq 0 .
\end{aligned}
$$

Definition 2.2. Let $E$ be a subset of $[a, c] \times \mathbb{R}^{n}$. We say that $f\left(t, x_{0}, x_{1}, \ldots, x_{n-1}\right)$ satisfies the Nagumo condition on $E$ if there exists a continuous function $\phi:[0,+\infty) \rightarrow(0,+\infty)$ such that

$$
\begin{gathered}
\left|f\left(t, x_{0}, x_{1}, \ldots, x_{n-1}\right)\right| \leq \phi\left(\left|x_{n-1}\right|\right), \quad\left(t, x_{0}, x_{1}, \ldots, x_{n-1}\right) \in E, \\
\int_{0}^{+\infty} \frac{s d s}{\phi(s)}=+\infty .
\end{gathered}
$$

Lemma 2.3 (see [10]). Let $f:[a, c] \times \mathbb{R}^{n} \rightarrow \mathbb{R}$ be a continuous function satisfying the Nagumo condition on

$$
E=\left\{\left(t, x_{0}, x_{1}, \ldots, x_{n-1}\right) \in[a, c] \times \mathbb{R}^{n}: \gamma_{i}(t) \leq x_{i} \leq \Gamma_{i}(t), i=0,1, \ldots, n-2\right\},
$$


where $\gamma_{i}(t), \Gamma_{i}(t):[a, c] \rightarrow \mathbb{R}$ are continuous functions such that

$$
\gamma_{i}(t) \leq \Gamma_{i}(t), \quad i=0,1, \ldots, n-2, t \in[a, c] .
$$

Then there exists a constant $r>0$ (depending only on $\gamma_{n-2}(t), \Gamma_{n-2}(t)$ and $\phi(t)$ ) such that every solution $x(t)$ of (1.1) with

$$
\gamma_{i}(t) \leq x^{(i)}(t) \leq \Gamma_{i}(t), \quad i=0,1, \ldots, n-2, t \in[a, c]
$$

satisfies $\left\|x^{(n-1)}\right\|_{\infty} \leq r$.

Lemma 2.4. Let $\phi:[0,+\infty) \rightarrow(0,+\infty)$ be a continuous function. Then boundary value problem

$$
\begin{gathered}
x^{(n)}=x^{(n-2)} \phi\left(\left|x^{(n-1)}\right|\right), \quad t \in[a, c], \\
x^{(n-2)}(a)=x^{(i)}(b)=x^{(n-2)}(c)=0, \quad i=0,1, \ldots, n-3
\end{gathered}
$$

has only the trivial solution.

Proof. Suppose that $x_{0}(t)$ is a nontrivial solution of BVP (2.6), (2.7). Then there exists $t_{0} \in$ $(a, c)$ such that $x_{0}^{(n-2)}\left(t_{0}\right)>0$ or $x_{0}^{(n-2)}\left(t_{0}\right)<0$. We may assume $x_{0}^{(n-2)}\left(t_{0}\right)>0$. There exists $t_{1} \in(a, c)$ such that

$$
\max _{t \in[a, c]} x_{0}^{(n-2)}(t):=x_{0}^{(n-2)}\left(t_{1}\right)>0
$$

Then $x_{0}^{(n-1)}\left(t_{1}\right)=0, x_{0}^{(n)}\left(t_{1}\right) \leq 0$. From (2.6) we have

$$
0 \geq x_{0}^{(n)}\left(t_{1}\right)=x_{0}^{(n-2)}\left(t_{1}\right) \phi\left(\left|x_{0}^{(n-1)}\left(t_{1}\right)\right|\right)>0,
$$

which is a contradiction. Hence BVP (2.6), (2.7) has only the trivial solution.

\section{Main Results}

We may now formulate and prove our main results on the existence and uniqueness of solutions for $n$ th-order three point boundary value problem (1.1), (1.2).

Theorem 3.1. Assume that

(i) there exist lower and upper solutions $\alpha(t), \beta(t)$ of $B V P(1.1),(1.2)$, respectively, such that

$$
\begin{aligned}
(-1)^{n-i} \alpha^{(i)}(t) & \leq(-1)^{n-i} \beta^{(i)}(t), \quad t \in[a, b], i=0,1, \ldots, n-2, \\
\alpha^{(i)}(t) & \leq \beta^{(i)}(t), \quad t \in[b, c], i=0,1, \ldots, n-2 ;
\end{aligned}
$$


(ii) $f\left(t, x_{0}, \ldots, x_{n-1}\right)$ is continuous on $[a, c] \times \mathbb{R}^{n},(-1)^{n-i} f\left(t, x_{0}, \ldots, x_{n-1}\right)$ is nonincreasing in $x_{i}(i=0,1, \ldots, n-3)$ on $D_{a^{\prime}}^{b}$ and $f\left(t, x_{0}, \ldots, x_{n-1}\right)$ is nonincreasing in $x_{i}(i=0,1, \ldots, n-$ 3) on $D_{b}^{c}$ and satisfies the Nagumo condition on $D_{a}^{c}$, where

$$
\begin{aligned}
\varphi_{i}(t) & =\min \left\{\alpha^{(i)}(t), \beta^{(i)}(t)\right\}, \quad \varphi_{i}(t)=\max \left\{\alpha^{(i)}(t), \beta^{(i)}(t)\right\}, \quad i=0, \ldots, n-2, \\
D_{a}^{b} & =\left\{\left(t, x_{0}, \ldots, x_{n-1}\right) \in[a, b] \times \mathbb{R}^{n}: \varphi_{i}(t) \leq x_{i} \leq \psi_{i}(t), i=0, \ldots, n-2\right\}, \\
D_{b}^{c} & =\left\{\left(t, x_{0}, \ldots, x_{n-1}\right) \in[b, c] \times \mathbb{R}^{n}: \varphi_{i}(t) \leq x_{i} \leq \psi_{i}(t), i=0, \ldots, n-2\right\}, \\
D_{a}^{c} & =\left\{\left(t, x_{0}, \ldots, x_{n-1}\right) \in[a, c] \times \mathbb{R}^{n}: \varphi_{i}(t) \leq x_{i} \leq \psi_{i}(t), i=0, \ldots, n-2\right\} ;
\end{aligned}
$$

(iii) $g\left(x_{0}, x_{1}, \ldots, x_{n-1}\right)$ is continuous on $\mathbb{R}^{n}$, and $(-1)^{n-i} g\left(x_{0}, x_{1}, \ldots, x_{n-1}\right)$ is nonincreasing in $x_{i}(i=0,1, \ldots, n-3)$ and nondecreasing in $x_{n-1}$ on $\prod_{i=0}^{n-2}\left[\varphi_{i}(a), \psi_{i}(a)\right] \times \mathbb{R}$;

(iv) $h\left(x_{0}, x_{1}, \ldots, x_{n-1}\right)$ is continuous on $\mathbb{R}^{n}$, and nonincreasing in $x_{i}(i=0,1, \ldots, n-3)$ and nondecreasing in $x_{n-1}$ on $\prod_{i=0}^{n-2}\left[\varphi_{i}(c), \psi_{i}(c)\right] \times \mathbb{R}$.

Then BVP (1.1), (1.2) has at least one solution $x(t) \in C^{n}[a, c]$ such that for each $i=0,1, \ldots, n-2$,

$$
\begin{gathered}
(-1)^{n-i} \alpha^{(i)}(t) \leq(-1)^{n-i} x^{(i)}(t) \leq(-1)^{n-i} \beta^{(i)}(t), \quad t \in[a, b] \\
\alpha^{(i)}(t) \leq x^{(i)}(t) \leq \beta^{(i)}(t), \quad t \in[b, c] .
\end{gathered}
$$

Proof. For each $i=0,1, \ldots, n-2$ define

$$
w_{i}(t, x)= \begin{cases}\psi_{i}(t), & x>\psi_{i}(t) \\ x, & \varphi_{i}(t) \leq x \leq \psi_{i}(t) \\ \varphi_{i}(t), & x<\varphi_{i}(t)\end{cases}
$$

where $\varphi_{i}(t)=\min \left\{\alpha^{(i)}(t), \beta^{(i)}(t)\right\}, \psi_{i}(t)=\max \left\{\alpha^{(i)}(t), \beta^{(i)}(t)\right\}$.

For $\lambda \in[0,1]$, we consider the auxiliary equation

$$
\begin{aligned}
x^{(n)}(t)= & \lambda f\left(t, w_{0}(t, x(t)), \ldots, w_{n-2}\left(t, x^{(n-2)}(t)\right), x^{(n-1)}(t)\right) \\
& +\left[x^{(n-2)}(t)-\lambda w_{n-2}\left(t, x^{(n-2)}(t)\right)\right] \phi\left(\left|x^{(n-1)}(t)\right|\right),
\end{aligned}
$$

where $\phi$ is given by the Nagumo condition, with the boundary conditions

$$
\begin{aligned}
x^{(n-2)}(a)=\lambda\left[w_{n-2}\left(a, x^{(n-2)}(a)\right)-g\left(w_{0}(a, x(a)), \ldots, w_{n-2}\left(a, x^{(n-2)}(a)\right), x^{(n-1)}(a)\right)\right], \\
x^{(i)}(b)=\lambda \mu_{i}, \quad i=0,1, \ldots, n-3, \\
x^{(n-2)}(c)=\lambda\left[w_{n-2}\left(c, x^{(n-2)}(c)\right)-h\left(w_{0}(c, x(c)), \ldots, w_{n-2}\left(c, x^{(n-2)}(c)\right), x^{(n-1)}(c)\right)\right] .
\end{aligned}
$$


Then we can choose a constant $M_{n-2}>0$ such that

$$
\begin{aligned}
& -M_{n-2}<\alpha^{(n-2)}(t) \leq \beta^{(n-2)}(t)<M_{n-2}, \quad t \in[a, c], \\
& f\left(t, \alpha(t), \ldots, \alpha^{(n-2)}(t), 0\right)-\left[M_{n-2}+\alpha^{(n-2)}(t)\right] \phi(0)<0, \quad t \in[a, c], \\
& f\left(t, \beta(t), \ldots, \beta^{(n-2)}(t), 0\right)+\left[M_{n-2}-\beta^{(n-2)}(t)\right] \phi(0)>0, \quad t \in[a, c], \\
& \left|\alpha^{(n-2)}(a)-g\left(\alpha(a), \ldots, \alpha^{(n-2)}(a), 0\right)\right|<M_{n-2}, \\
& \left|\beta^{(n-2)}(a)-g\left(\beta(a), \ldots, \beta^{(n-2)}(a), 0\right)\right|<M_{n-2}, \\
& \left|\alpha^{(n-2)}(c)-h\left(\alpha(c), \ldots, \alpha^{(n-2)}(c), 0\right)\right|<M_{n-2}, \\
& \left|\beta^{(n-2)}(c)-h\left(\beta(c), \ldots, \beta^{(n-2)}(c), 0\right)\right|<M_{n-2} .
\end{aligned}
$$

In the following, we will complete the proof in four steps.

Step 1. Show that every solution $x(t)$ of BVP (3.5), (3.6) satisfies

$$
\left|x^{(n-2)}(t)\right|<M_{n-2}, \quad t \in[a, c],
$$

independently of $\lambda \in[0,1]$.

Suppose that the estimate $\left|x^{(n-2)}(t)\right|<M_{n-2}$ is not true. Then there exists $t_{0} \in[a, c]$ such that $x^{(n-2)}\left(t_{0}\right) \geq M_{n-2}$ or $x^{(n-2)}\left(t_{0}\right) \leq-M_{n-2}$. We may assume $x^{(n-2)}\left(t_{0}\right) \geq M_{n-2}$. There exists $t_{1} \in[a, c]$ such that

$$
\max _{t \in[a, c]} x^{(n-2)}(t):=x^{(n-2)}\left(t_{1}\right)\left(\geq M_{n-2}>0\right) .
$$

There are three cases to consider.

Case $1\left(t_{1} \in(a, c)\right)$. In this case, $x^{(n-1)}\left(t_{1}\right)=0$ and $x^{(n)}\left(t_{1}\right) \leq 0$. For $\lambda \in(0,1]$, by (3.8), we get the following contradiction:

$$
\begin{aligned}
0 \geq & x^{(n)}\left(t_{1}\right) \\
= & \lambda f\left(t_{1}, w_{0}\left(t_{1}, x\left(t_{1}\right)\right), \ldots, w_{n-2}\left(t_{1}, x^{(n-2)}\left(t_{1}\right)\right), x^{(n-1)}\left(t_{1}\right)\right) \\
& +\left[x^{(n-2)}\left(t_{1}\right)-\lambda w_{n-2}\left(t_{1}, x^{(n-2)}\left(t_{1}\right)\right)\right] \phi\left(\left|x^{(n-1)}\left(t_{1}\right)\right|\right) \\
= & \lambda f\left(t_{1}, w_{0}\left(t_{1}, x\left(t_{1}\right)\right), \ldots, w_{n-3}\left(t_{1}, x^{(n-3)}\left(t_{1}\right)\right), \beta^{(n-2)}\left(t_{1}\right), 0\right) \\
& +\left[x^{(n-2)}\left(t_{1}\right)-\lambda \beta^{(n-2)}\left(t_{1}\right)\right] \phi(0) \\
\geq & \lambda\left\{f\left(t_{1}, \beta\left(t_{1}\right), \ldots, \beta^{(n-2)}\left(t_{1}\right), 0\right)+\left[M_{n-2}-\beta^{(n-2)}\left(t_{1}\right)\right] \phi(0)\right\}>0,
\end{aligned}
$$


and for $\mathcal{\lambda}=0$, we have the following contradiction:

$$
0 \geq x^{(n)}\left(t_{1}\right)=x^{(n-2)}\left(t_{1}\right) \phi(0) \geq M_{n-2} \phi(0)>0 .
$$

Case $2\left(t_{1}=a\right)$. In this case,

$$
\max _{t \in[a, c]} x^{(n-2)}(t):=x^{(n-2)}(a)\left(\geq M_{n-2}>0\right),
$$

and $x^{(n-1)}(a) \leq 0$. For $\lambda=0$, by (3.6) we have the following contradiction:

$$
0<M_{n-2} \leq x^{(n-2)}(a)=0 .
$$

For $\lambda \in(0,1]$, by (3.9) and condition (iii) we can get the following contradiction:

$$
\begin{aligned}
M_{n-2} & \leq x^{(n-2)}(a), \\
& =\lambda\left[w_{n-2}\left(a, x^{(n-2)}(a)\right)-g\left(w_{0}(a, x(a)), \ldots, w_{n-2}\left(a, x^{(n-2)}(a)\right), x^{(n-1)}(a)\right)\right], \\
& \leq \lambda\left[\beta^{(n-2)}(a)-g\left(\beta(a), \ldots, \beta^{(n-2)}(a), 0\right)\right]<M_{n-2} .
\end{aligned}
$$

Case $3\left(t_{1}=c\right)$. In this case,

$$
\max _{t \in[a, c]} x^{(n-2)}(t):=x^{(n-2)}(c)\left(\geq M_{n-2}>0\right),
$$

and $x^{(n-1)}(c) \geq 0$. For $\lambda=0$, by (3.6) we have the following contradiction:

$$
0<M_{n-2} \leq x^{(n-2)}(c)=0 .
$$

For $\lambda \in(0,1]$, by (3.10) and condition (iv) we can get the following contradiction:

$$
\begin{aligned}
M_{n-2} & \leq x^{(n-2)}(c), \\
& =\lambda\left[w_{n-2}\left(c, x^{(n-2)}(c)\right)-h\left(w_{0}(c, x(c)), \ldots, w_{n-2}\left(c, x^{(n-2)}(c)\right), x^{(n-1)}(c)\right)\right] \\
& \leq \lambda\left[\beta^{(n-2)}(c)-h\left(\beta(c), \ldots, \beta^{(n-2)}(c), 0\right)\right]<M_{n-2} .
\end{aligned}
$$

By (3.6), the estimates

$$
\left|x^{(i)}(t)\right|<M_{i}:=(c-a) M_{i+1}+\left|\mu_{i}\right|, \quad i=0,1, \ldots, n-3, t \in[a, c]
$$

are obtained by integration. 
Step 2. Show that there exists $M_{n-1}>0$ such that every solution $x(t)$ of BVP (3.5), (3.6) satisfies

$$
\left|x^{(n-1)}(t)\right|<M_{n-1}, \quad t \in[a, c]
$$

independently of $\lambda \in[0,1]$.

Let

$$
E=\left\{\left(t, x_{0}, \ldots, x_{n-1}\right) \in[a, c] \times \mathbb{R}^{n}:\left|x_{i}\right| \leq M_{i}, i=0,1, \ldots, n-2\right\},
$$

and define the function $F_{\lambda}:[a, c] \times \mathbb{R}^{n} \rightarrow \mathbb{R}$ as follows:

$$
\begin{aligned}
F_{\lambda}\left(t, x_{0}, \ldots, x_{n-1}\right)= & \lambda f\left(t, w_{0}\left(t, x_{0}\right), \ldots, w_{n-2}\left(t, x_{n-2}\right), x_{n-1}\right) \\
& +\left[x_{n-2}-\lambda w_{n-2}\left(t, x_{n-2}\right)\right] \phi\left(\left|x_{n-1}\right|\right) .
\end{aligned}
$$

In the following, we show that $F_{\lambda}\left(t, x_{0}, \ldots, x_{n-1}\right)$ satisfies the Nagumo condition on $E$, independently of $\lambda \in[0,1]$. In fact, since $f$ satisfies the Nagumo condition on $D_{a}^{c}$, we have

$$
\begin{aligned}
\left|F_{\curlywedge}\left(t, x_{0}, \ldots, x_{n-1}\right)\right|= & \mid \lambda f\left(t, w_{0}\left(t, x_{0}\right), \ldots, w_{n-2}\left(t, x_{n-2}\right), x_{n-1}\right) \\
& +\left[x_{n-2}-\lambda w_{n-2}\left(t, x_{n-2}\right)\right] \phi\left(\left|x_{n-1}\right|\right) \mid \\
\leq & {\left[1+2 M_{n-2}\right] \phi\left(\left|x_{n-1}\right|\right):=\phi_{E}\left(\left|x_{n-1}\right|\right) . }
\end{aligned}
$$

Furthermore, we obtain

$$
\int_{0}^{+\infty} \frac{s}{\phi_{E}(s)} d s=\int_{0}^{+\infty} \frac{s}{\left(1+2 M_{n-2}\right) \phi(s)} d s=+\infty
$$

Thus, $F_{\lambda}$ satisfies the Nagumo condition on $E$, independently of $\lambda \in[0,1]$. Let

$$
\gamma_{i}(t)=-M_{i}, \quad \Gamma_{i}(t)=M_{i}, \quad i=0,1, \ldots, n-2, t \in[a, c]
$$

By Step 1 and Lemma 2.3, there exists $M_{n-1}>0$ such that $\left|x^{(n-1)}(t)\right|<M_{n-1}$ for $t \in[a, c]$. Since $M_{n-2}$ and $\phi_{E}$ do not depend on $\lambda$, the estimate $\left|x^{(n-1)}(t)\right|<M_{n-1}$ on $[a, c]$ is also independent of $\lambda$.

Step 3. Show that for $\lambda=1$, BVP (3.5), (3.6) has at least one solution $x_{1}(t)$.

Define the operators as follows:

$$
L: C^{n}[a, c] \subset C^{n-1}[a, c] \longrightarrow C[a, c] \times \mathbb{R}^{n},
$$


by

$$
\begin{gathered}
L x=\left(x^{(n)}(t), x^{(n-2)}(a), x(b), \ldots, x^{(n-3)}(b), x^{(n-2)}(c)\right), \\
N_{\lambda}: C^{n-1}[a, c] \longrightarrow C[a, c] \times \mathbb{R}^{n},
\end{gathered}
$$

by

$$
N_{\curlywedge} x=\left(F_{\curlywedge}\left(t, x(t), \ldots, x^{(n-1)}(t)\right), A_{\curlywedge}, \lambda \mu_{0}, \ldots, \lambda \mu_{n-3}, C_{\lambda}\right)
$$

with

$$
\begin{aligned}
A_{\curlywedge} & :=\lambda\left[w_{n-2}\left(a, x^{(n-2)}(a)\right)-g\left(w_{0}(a, x(a)), \ldots, w_{n-2}\left(a, x^{(n-2)}(a)\right), x^{(n-1)}(a)\right)\right] \\
C_{\lambda} & :=\lambda\left[w_{n-2}\left(c, x^{(n-2)}(c)\right)-h\left(w_{0}(c, x(c)), \ldots, w_{n-2}\left(c, x^{(n-2)}(c)\right), x^{(n-1)}(c)\right)\right] .
\end{aligned}
$$

Since $L^{-1}$ is compact, we have the following compact operator:

$$
T_{\lambda}: C^{n-1}[a, c] \longrightarrow C^{n-1}[a, c]
$$

defined by

$$
T_{\lambda}(x)=L^{-1} N_{\lambda}(x)
$$

Consider the set $\Omega=\left\{x \in C^{n-1}[a, c]:\left\|x^{(i)}\right\|_{\infty}<M_{i}, i=0,1, \ldots, n-1\right\}$.

By Steps 1 and 2 , the degree $\operatorname{deg}\left(I-T_{\lambda}, \Omega, 0\right)$ is well defined for every $\lambda \in[0,1]$, and by homotopy invariance, we get

$$
\operatorname{deg}\left(I-T_{0}, \Omega, 0\right)=\operatorname{deg}\left(I-T_{1}, \Omega, 0\right) .
$$

Since the equation $x=T_{0}(x)$ has only the trivial solution from Lemma 2.4, by the degree theory we have

$$
\operatorname{deg}\left(I-T_{1}, \Omega, 0\right)=\operatorname{deg}\left(I-T_{0}, \Omega, 0\right)= \pm 1 .
$$

Hence, the equation $x=T_{1}(x)$ has at least one solution. That is, the boundary value problem

$$
\begin{aligned}
x^{(n)}(t)= & f\left(t, w_{0}(t, x(t)), \ldots, w_{n-2}\left(t, x^{(n-2)}(t)\right), x^{(n-1)}(t)\right) \\
& +\left[x^{(n-2)}(t)-w_{n-2}\left(t, x^{(n-2)}(t)\right)\right] \phi\left(\left|x^{(n-1)}(t)\right|\right),
\end{aligned}
$$


with the boundary conditions

$$
\begin{gathered}
x^{(n-2)}(a)=w_{n-2}\left(a, x^{(n-2)}(a)\right)-g\left(w_{0}(a, x(a)), \ldots, w_{n-2}\left(a, x^{(n-2)}(a)\right), x^{(n-1)}(a)\right), \\
x^{(i)}(b)=\mu_{i}, \quad i=0,1, \ldots, n-3, \\
x^{(n-2)}(c)=w_{n-2}\left(c, x^{(n-2)}(c)\right)-h\left(w_{0}(c, x(c)), \ldots, w_{n-2}\left(c, x^{(n-2)}(c)\right), x^{(n-1)}(c)\right),
\end{gathered}
$$

has at least one solution $x_{1}(t)$ in $\Omega$.

Step 4. Show that $x_{1}(t)$ is a solution of BVP (1.1), (1.2).

In fact, the solution $x_{1}(t)$ of BVP (3.36), (3.37) will be a solution of BVP (1.1), (1.2), if it satisfies

$$
\varphi_{i}(t) \leq x_{1}^{(i)}(t) \leq \psi_{i}(t), \quad i=0,1, \ldots, n-2, t \in[a, c]
$$

By contradiction, suppose that there exists $t_{0} \in[a, c]$ such that $x_{1}^{(n-2)}\left(t_{0}\right)>\psi_{n-2}\left(t_{0}\right)$. There exists $t_{1} \in[a, c]$ such that

$$
\max _{t \in[a, c]}\left[x_{1}^{(n-2)}(t)-\psi_{n-2}(t)\right]:=x_{1}^{(n-2)}\left(t_{1}\right)-\psi_{n-2}\left(t_{1}\right)>0
$$

Now there are three cases to consider.

Case $1\left(t_{1} \in(a, c)\right)$. In this case, since $\psi_{n-2}(t)=\beta^{(n-2)}(t)$ on $[a, c]$, we have $x_{1}^{(n-1)}\left(t_{1}\right)=\beta^{(n-1)}\left(t_{1}\right)$ and $x_{1}^{(n)}\left(t_{1}\right) \leq \beta^{(n)}\left(t_{1}\right)$. By conditions (i) and (ii), we get the following contradiction:

$$
\begin{aligned}
0 \geq & x_{1}^{(n)}\left(t_{1}\right)-\beta^{(n)}\left(t_{1}\right) \\
\geq & f\left(t_{1}, w_{0}\left(t_{1}, x_{1}\left(t_{1}\right)\right), \ldots, w_{n-2}\left(t_{1}, x_{1}^{(n-2)}\left(t_{1}\right)\right), x_{1}^{(n-1)}\left(t_{1}\right)\right) \\
& +\left[x_{1}^{(n-2)}\left(t_{1}\right)-w_{n-2}\left(t_{1}, x_{1}^{(n-2)}\left(t_{1}\right)\right)\right] \phi\left(\left|x_{1}^{(n-1)}\left(t_{1}\right)\right|\right)-f\left(t_{1}, \beta\left(t_{1}\right), \ldots, \beta^{(n-1)}\left(t_{1}\right)\right) \\
\geq & f\left(t_{1}, \beta\left(t_{1}\right), \ldots, \beta^{(n-1)}\left(t_{1}\right)\right)+\left[x_{1}^{(n-2)}\left(t_{1}\right)-\beta^{(n-2)}\left(t_{1}\right)\right] \phi\left(\left|x_{1}^{(n-1)}\left(t_{1}\right)\right|\right) \\
& -f\left(t_{1}, \beta\left(t_{1}\right), \ldots, \beta^{(n-1)}\left(t_{1}\right)\right) \\
= & {\left[x_{1}^{(n-2)}\left(t_{1}\right)-\beta^{(n-2)}\left(t_{1}\right)\right] \phi\left(\left|x_{1}^{(n-1)}\left(t_{1}\right)\right|\right)>0 . }
\end{aligned}
$$

Case $2\left(t_{1}=a\right)$. In this case, we have

$$
\max _{t \in[a, c]}\left[x_{1}^{(n-2)}(t)-\psi_{n-2}(t)\right]:=x_{1}^{(n-2)}(a)-\beta^{(n-2)}(a)>0
$$


and $x_{1}^{(n-1)}(a) \leq \beta^{(n-1)}(a)$. By (3.37) and conditions (i) and (iii) we can get the following contradiction:

$$
\begin{aligned}
\beta^{(n-2)}(a) & <x_{1}^{(n-2)}(a), \\
& =w_{n-2}\left(a, x_{1}^{(n-2)}(a)\right)-g\left(w_{0}\left(a, x_{1}(a)\right), \ldots, w_{n-2}\left(a, x_{1}^{(n-2)}(a)\right), x_{1}^{(n-1)}(a)\right) \\
& \leq \beta^{(n-2)}(a)-g\left(\beta(a), \ldots, \beta^{(n-2)}(a), \beta^{(n-1)}(a)\right) \leq \beta^{(n-2)}(a) .
\end{aligned}
$$

Case $3\left(t_{1}=c\right)$. In this case, we have

$$
\max _{t \in[a, c]}\left[x_{1}^{(n-2)}(t)-\psi_{n-2}(t)\right]:=x_{1}^{(n-2)}(c)-\beta^{(n-2)}(c)>0,
$$

and $x_{1}^{(n-1)}(c) \geq \beta^{(n-1)}(c)$. By (3.37) and conditions (i) and (iv) we can get the following contradiction:

$$
\begin{aligned}
\beta^{(n-2)}(c) & <x_{1}^{(n-2)}(c) \\
& =w_{n-2}\left(c, x_{1}^{(n-2)}(c)\right)-h\left(w_{0}\left(c, x_{1}(c)\right), \ldots, w_{n-2}\left(c, x_{1}^{(n-2)}(c)\right), x_{1}^{(n-1)}(c)\right) \\
& \leq \beta^{(n-2)}(c)-h\left(\beta(c), \ldots, \beta^{(n-2)}(c), \beta^{(n-1)}(c)\right) \leq \beta^{(n-2)}(c) .
\end{aligned}
$$

Similarly, we can show that $\varphi_{n-2}(t) \leq x_{1}^{(n-2)}(t)$ on $[a, c]$. Hence

$$
\alpha^{(n-2)}(t)=\varphi_{n-2}(t) \leq x_{1}^{(n-2)}(t) \leq \psi_{n-2}(t)=\beta^{(n-2)}(t), \quad t \in[a, c] .
$$

Also, by boundary condition (3.37) and condition (i), we have

$$
\begin{aligned}
& \alpha^{(i)}(b)=x_{1}^{(i)}(b)=\beta^{(i)}(b), \quad i=n-1-2 j, j=1,2, \ldots,\left[\frac{n-1}{2}\right], \\
& \alpha^{(i)}(b) \leq x_{1}^{(i)}(b) \leq \beta^{(i)}(b), \quad i=n-2-2 j, j=1,2, \ldots,\left[\frac{n-2}{2}\right] .
\end{aligned}
$$

Therefore by integration we have for each $i=0,1, \ldots, n-2$,

$$
\begin{gathered}
(-1)^{n-i} \alpha^{(i)}(t) \leq(-1)^{n-i} x_{1}^{(i)}(t) \leq(-1)^{n-i} \beta^{(i)}(t), \quad t \in[a, b], \\
\alpha^{(i)}(t) \leq x_{1}^{(i)}(t) \leq \beta^{(i)}(t), \quad t \in[b, c],
\end{gathered}
$$


that is,

$$
\varphi_{i}(t) \leq x_{1}^{(i)}(t) \leq \psi_{i}(t), \quad i=0,1, \ldots, n-2, t \in[a, c]
$$

Hence $x_{1}(t)$ is a solution of BVP (1.1), (1.2) and satisfies (3.3).

Now we give a uniqueness theorem by assuming additionally the differentiability for functions $f, g$ and $h$, and a kind of estimating condition in Theorem 3.1.

Theorem 3.2. Assume that

(i) there exist lower and upper solutions $\alpha(t), \beta(t)$ of $B V P(1.1),(1.2)$, respectively, such that

$$
\begin{aligned}
(-1)^{n-i} \alpha^{(i)}(t) & \leq(-1)^{n-i} \beta^{(i)}(t), \quad t \in[a, b], i=0,1, \ldots, n-2, \\
\alpha^{(i)}(t) & \leq \beta^{(i)}(t), \quad t \in[b, c], i=0,1, \ldots, n-2 ;
\end{aligned}
$$

(ii) $f\left(t, x_{0}, \ldots, x_{n-1}\right)$ and its first-order partial derivatives in $x_{i}(i=0,1, \ldots, n-1)$ are continuous on $[a, c] \times \mathbb{R}^{n},(-1)^{n-i}\left(\partial f / \partial x_{i}\right) \leq 0(i=0,1, \ldots, n-3)$ on $D_{a}^{b}, \partial f / \partial x_{i} \leq$ $0(i=0,1, \ldots, n-3)$ on $D_{b}^{c}$ and satisfy the Nagumo condition on $D_{a}^{c}$;

(iii) $g\left(x_{0}, x_{1}, \ldots, x_{n-1}\right)$ is continuous on $\mathbb{R}^{n}$ and continuously partially differentiable on $\prod_{i=0}^{n-2}\left[\varphi_{i}(a), \psi_{i}(a)\right] \times \mathbb{R}$, and

$$
\begin{gathered}
(-1)^{n-i} \frac{\partial g}{\partial x_{i}} \leq 0, \quad i=0,1, \ldots, n-3 \\
\frac{\partial g}{\partial x_{n-1}} \leq 0, \quad \text { on } \prod_{i=0}^{n-2}\left[\varphi_{i}(a), \psi_{i}(a)\right] \times \mathbb{R}
\end{gathered}
$$

(iv) $h\left(x_{0}, x_{1}, \ldots, x_{n-1}\right)$ is continuous on $\mathbb{R}^{n}$ and continuously partially differentiable on $\prod_{i=0}^{n-2}\left[\varphi_{i}(c), \psi_{i}(c)\right] \times \mathbb{R}$, and

$$
\begin{gathered}
\frac{\partial h}{\partial x_{i}} \leq 0, \quad i=0,1, \ldots, n-3 \\
\frac{\partial h}{\partial x_{n-1}} \geq 0, \quad \text { on } \prod_{i=0}^{n-2}\left[\varphi_{i}(c), \psi_{i}(c)\right] \times \mathbb{R}
\end{gathered}
$$


(v) there exists a function $\gamma(t) \in C^{n}[a, c]$ such that $\gamma^{(n-2)}(t)>0$ on $[a, c]$, and

$$
\begin{gathered}
\gamma^{(n)}(t)<\sum_{i=0}^{n-1} \frac{\partial f}{\partial x_{i}} \cdot \gamma^{(i)}(t), \quad \text { on } D_{a}^{c} \\
\sum_{i=0}^{n-1} \frac{\partial g}{\partial x_{i}} \cdot \gamma^{(i)}(a)>0, \quad \text { on } \prod_{i=0}^{n-2}\left[\varphi_{i}(a), \psi_{i}(a)\right] \times \mathbb{R}, \\
\sum_{i=0}^{n-1} \frac{\partial h}{\partial x_{i}} \cdot \gamma^{(i)}(c)>0, \quad \text { on } \prod_{i=0}^{n-2}\left[\varphi_{i}(c), \psi_{i}(c)\right] \times \mathbb{R}, \\
\gamma^{(i)}(b)=0, \quad \text { if } n-i: \text { odd }, i=0,1, \ldots, n-3, \\
\gamma^{(i)}(b) \geq 0, \quad \text { if } n-i: \text { even, } i=0,1, \ldots, n-3 .
\end{gathered}
$$

Then BVP (1.1), (1.2) has a unique solution $x(t)$ satisfying (3.3).

Proof. The existence of a solution for BVP (1.1), (1.2) satisfying (3.3) follows from Theorem 3.1.

Now, we prove the uniqueness of solution for BVP (1.1), (1.2). To do this, we let $x_{1}(t)$ and $x_{2}(t)$ are any two solutions of BVP (1.1), (1.2) satisfying (3.3). Let $z(t)=x_{2}(t)-x_{1}(t)$. It is easy to show that $z(t)$ is a solution of the following boundary value problem

$$
\begin{gathered}
z^{(n)}(t)=\sum_{i=0}^{n-1} d_{i}(t) z^{(i)}(t), \\
\sum_{i=0}^{n-1} a_{i} z^{(i)}(a)=0, \quad \sum_{i=0}^{n-1} c_{i} z^{(i)}(c)=0, \\
z^{(i)}(b)=0, \quad i=0,1, \ldots, n-3,
\end{gathered}
$$

where for each $i=0,1, \ldots, n-1$,

$$
\begin{aligned}
d_{i}(t) & =\int_{0}^{1} \frac{\partial}{\partial x_{i}} f\left(t, x_{1}(t)+\theta z(t), x_{1}^{\prime}(t)+\theta z^{\prime}(t), \ldots, x_{1}^{(n-1)}(t)+\theta z^{(n-1)}(t)\right) d \theta \\
a_{i} & =\int_{0}^{1} \frac{\partial}{\partial x_{i}} g\left(x_{1}(a)+\theta z(a), x_{1}^{\prime}(a)+\theta z^{\prime}(a), \ldots, x_{1}^{(n-1)}(a)+\theta z^{(n-1)}(a)\right) d \theta \\
c_{i} & =\int_{0}^{1} \frac{\partial}{\partial x_{i}} h\left(x_{1}(c)+\theta z(c), x_{1}^{\prime}(c)+\theta z^{\prime}(c), \ldots, x_{1}^{(n-1)}(c)+\theta z^{(n-1)}(c)\right) d \theta
\end{aligned}
$$


By conditions (ii), (iii), and (iv), we have that $d_{i}(t) \in C[a, c], i=0,1, \ldots, n-3$, and

$$
\begin{gathered}
(-1)^{n-i} d_{i}(t) \leq 0, \quad i=0,1, \ldots, n-3, t \in[a, b] \\
d_{i}(t) \leq 0, \quad i=0,1, \ldots, n-3, t \in[b, c] \\
(-1)^{n-i} a_{i} \leq 0, \quad i=0,1, \ldots, n-3, a_{n-1} \leq 0 \\
c_{i} \leq 0, \quad i=0,1, \ldots, n-3, c_{n-1} \geq 0 .
\end{gathered}
$$

Now suppose that there exists $t_{0} \in[a, c]$ such that $z^{(n-2)}\left(t_{0}\right) \neq 0$. Without loss of generality assume $z^{(n-2)}\left(t_{0}\right)>0$, and let

$$
\Omega=\left\{M: M z^{(n-2)}(t)<\gamma^{(n-2)}(t), t \in[a, c]\right\}
$$

It is easy to see that $0 \in \Omega$ by condition (v), hence $\Omega \neq \emptyset$. Let $M_{0}=\sup \Omega$. We have that $0<M_{0}<+\infty, M_{0} z^{(n-2)}(t) \leq \gamma^{(n-2)}(t)$ on $[a, c]$, and there exists a point $t_{1} \in[a, c]$ such that $M_{0} z^{(n-2)}\left(t_{1}\right)=\gamma^{(n-2)}\left(t_{1}\right)$. Furthermore $t_{1} \neq a, c$. In fact, if $t_{1}=a$, then $M_{0} z^{(n-1)}(a) \leq \gamma^{(n-1)}(a)$. By condition (v) and (3.55) we can easily show that

$$
(-1)^{n-i}\left[M_{0} z^{(i)}(t)-\gamma^{(i)}(t)\right] \leq 0, \quad i=0,1, \ldots, n-3, t \in[a, b]
$$

In particular

$$
(-1)^{n-i}\left[M_{0} z^{(i)}(a)-\gamma^{(i)}(a)\right] \leq 0, \quad i=0,1, \ldots, n-3 .
$$

Hence

$$
\sum_{i=0}^{n-1} M_{0} a_{i} z^{(i)}(a) \geq \sum_{i=0}^{n-1} a_{i} \gamma^{(i)}(a)>0,
$$

which contradicts to (3.54). Thus $t_{1} \neq a$. Similarly we can show that $t_{1} \neq c$. Consequently $M_{0} z^{(n-1)}\left(t_{1}\right)=\gamma^{(n-1)}\left(t_{1}\right)$.

Now, there are two cases to consider, that is

$$
t_{1} \in(a, b] \text { or } t_{1} \in[b, c) .
$$

If $t_{1} \in(a, b]$, then by (3.59) we have

$$
(-1)^{n-i}\left[M_{0} z^{(i)}\left(t_{1}\right)-\gamma^{(i)}\left(t_{1}\right)\right] \leq 0, \quad i=0,1, \ldots, n-3 .
$$


Thus, by (3.53) and condition (v) we have

$$
M_{0} z^{(n)}\left(t_{1}\right)=\sum_{i=0}^{n-1} M_{0} d_{i}\left(t_{1}\right) z^{(i)}\left(t_{1}\right) \geq \sum_{i=0}^{n-1} d_{i}\left(t_{1}\right) \gamma^{(i)}\left(t_{1}\right)>\gamma^{(n)}\left(t_{1}\right)
$$

Consequently, by Taylor's theorem there exists $t_{2} \in\left(t_{1}, c\right)$ such that

$$
M_{0} z^{(n-2)}(t)>\gamma^{(n-2)}(t), \quad \forall t \in\left(t_{1}, t_{2}\right]
$$

which is a contradiction.

A similar contradiction can be obtained if $t_{1} \in[b, c)$. Hence $z^{(n-2)}(t) \equiv 0$ on $[a, c]$. By (3.55), we obtain $z(t) \equiv 0$ on $[a, c]$. This completes the proof of the theorem.

Next we give two examples to demonstrate the application of Theorem 3.2.

Example 3.3. Consider the following third-order three point BVP:

$$
\begin{gathered}
x^{\prime \prime \prime}=-t x+\left(2 t^{2}+1\right) x^{\prime}+\frac{1}{3}\left(x^{\prime}\right)^{3}-t^{4} \sin \left(t+x^{\prime \prime}\right), t \in[-1,1], \\
1+3 x^{\prime}(-1)+\left(x^{\prime}(-1)\right)^{3}-\left(x^{\prime \prime}(-1)+1\right)^{3}=0, \\
x(0)=0, \\
-1-x(1)+2 x^{\prime}(1)+\left(x^{\prime}(1)\right)^{3}+\left(x^{\prime \prime}(1)+1\right)^{3}=0 .
\end{gathered}
$$

Let

$$
\begin{gathered}
f\left(t, x_{0}, x_{1}, x_{2}\right)=-t x_{0}+\left(2 t^{2}+1\right) x_{1}+\frac{1}{3} x_{1}^{3}-t^{4} \sin \left(t+x_{2}\right), \\
g\left(x_{0}, x_{1}, x_{2}\right)=1+3 x_{1}+x_{1}^{3}-\left(x_{2}+1\right)^{3}, \\
h\left(x_{0}, x_{1}, x_{2}\right)=-1-x_{0}+2 x_{1}+x_{1}^{3}+\left(x_{2}+1\right)^{3} .
\end{gathered}
$$

Choose $\alpha(t)=-t, \beta(t)=t$ and $\gamma(t)=t$. It is easy to check that $\alpha(t)=-t$, and $\beta(t)=t$ are lower and upper solutions of BVP (3.66), (3.67) respectively, and all the assumptions in Theorem 3.2 are satisfied. Therefore by Theorem 3.2 BVP (3.66), (3.67) has a unique solution $x=x(t)$ satisfying

$$
\begin{array}{cl}
t \leq x(t) \leq-t, \quad t \in[-1,0], \quad-t \leq x(t) \leq t, \quad t \in[0,1], \\
-1 \leq x^{\prime}(t) \leq 1, \quad t \in[-1,1] .
\end{array}
$$


Example 3.4. Consider the following fourth-order three point BVP:

$$
\begin{gathered}
x^{(4)}=-t^{2} x+x^{\prime \prime}+\left(x^{\prime \prime}\right)^{3}, \quad t \in[-1,1], \\
-x(-1)+\left(x^{\prime}(-1)\right)^{3}+13 x^{\prime \prime}(-1)=0, \\
x(0)=0, \quad x^{\prime}(0)=0, \\
-x(1)-4 x^{\prime}(1)+\left(x^{\prime}(1)\right)^{2}+9 x^{\prime \prime}(1)=0 .
\end{gathered}
$$

Let

$$
\begin{aligned}
& f\left(t, x_{0}, x_{1}, x_{2}, x_{3}\right)=-t^{2} x_{0}+x_{2}+x_{2}^{3} \\
& g\left(x_{0}, x_{1}, x_{2}, x_{3}\right)=-x_{0}+x_{1}^{3}+13 x_{2} \\
& h\left(x_{0}, x_{1}, x_{2}, x_{3}\right)=-x_{0}-4 x_{1}+x_{1}^{2}+9 x_{2} .
\end{aligned}
$$

Choose $\alpha(t)=-t^{2}, \beta(t)=t^{2}$ and $\gamma(t)=t^{2}$. It is easy to check that $\alpha(t)=-t^{2}$, and $\beta(t)=t^{2}$ are lower and upper solutions of BVP (3.70), (3.71), respectively, and all the assumptions in Theorem 3.2 are satisfied. Therefore by Theorem 3.2 BVP (3.70), (3.71) has a unique solution $x=x(t)$ satisfying

$$
\begin{aligned}
& -2 \leq x(t) \leq 2, \quad t \in[-1,1], \\
2 t \leq x^{\prime}(t) \leq-2 t, \quad t \in[-1,0], & -2 t \leq x^{\prime}(t) \leq 2 t, \quad t \in[0,1], \\
-t^{2} \leq x^{\prime \prime}(t) \leq t^{2}, & t \in[-1,1] .
\end{aligned}
$$

\section{References}

[1] A. R. Aftabizadeh, C. P. Gupta, and J.-M. Xu, "Existence and uniqueness theorems for three-point boundary value problems," SIAM Journal on Mathematical Analysis, vol. 20, no. 3, pp. 716-726, 1989.

[2] R. P. Agarwal, Boundary Value Problems for Higher Order Differential Equations, World Scientific, Teaneck, NJ, USA, 1986.

[3] R. P. Agarwal and F.-H. Wong, "Existence of positive solutions for non-positive higher-order BVPs," Journal of Computational and Applied Mathematics, vol. 88, no. 1, pp. 3-14, 1998.

[4] R. P. Agarwal, S. R. Grace, and D. O’Regan, "Semipositone higher-order differential equations," Applied Mathematics Letters, vol. 17, no. 2, pp. 201-207, 2004.

[5] A. Cabada, "The method of lower and upper solutions for second, third, fourth, and higher order boundary value problems," Journal of Mathematical Analysis and Applications, vol. 185, no. 2, pp. 302 320, 1994.

[6] A. Cabada, M. R. Grossinho, and F. Minhós, “Extremal solutions for third-order nonlinear problems with upper and lower solutions in reversed order," Nonlinear Analysis: Theory, Methods E Applications, vol. 62, no. 6, pp. 1109-1121, 2005.

[7] Z. Du, W. Ge, and X. Lin, "Existence of solutions for a class of third-order nonlinear boundary value problems," Journal of Mathematical Analysis and Applications, vol. 294, no. 1, pp. 104-112, 2004.

[8] Y. Feng and S. Liu, "Solvability of a third-order two-point boundary value problem," Applied Mathematics Letters, vol. 18, no. 9, pp. 1034-1040, 2005.

[9] M. R. Grossinho and F. M. Minhós, "Existence result for some third order separated boundary value problems," Nonlinear Analysis: Theory, Methods \& Applications, vol. 47, no. 4, pp. 2407-2418, 2001. 
[10] M. R. Grossinho and F. M. Minhós, “Upper and lower solutions for higher order boundary value problems," Nonlinear Studies, vol. 12, no. 2, pp. 165-176, 2005.

[11] M. R. Grossinho, F. M. Minhós, and A. I. Santos, "Solvability of some third-order boundary value problems with asymmetric unbounded nonlinearities," Nonlinear Analysis: Theory, Methods $\mathcal{E}$ Applications, vol. 62, no. 7, pp. 1235-1250, 2005.

[12] C. P. Gupta and V. Lakshmikantham, "Existence and uniqueness theorems for a third-order threepoint boundary value problem," Nonlinear Analysis: Theory, Methods E Applications, vol. 16, no. 11, pp. 949-957, 1991.

[13] J. Henderson and R. D. Taunton, "Solutions of boundary value problems by matching methods," Applicable Analysis, vol. 49, no. 3-4, pp. 235-246, 1993.

[14] Y. Lin and M. Pei, "Positive solutions for two-point semipositone right focal eigenvalue problem," Boundary Value Problems, vol. 2007, Article ID 23108, 12 pages, 2007.

[15] V. R. G. Moorti and J. B. Garner, "Existence-uniqueness theorems for three-point boundary value problems for nth-order nonlinear differential equations," Journal of Differential Equations, vol. 29, no. 2, pp. 205-213, 1978.

[16] K. N. Murty and Y. S. Rao, "A theory for existence and uniqueness of solutions to three-point boundary value problems," Journal of Mathematical Analysis and Applications, vol. 167, no. 1, pp. 43-48, 1992.

[17] M. Pei and S. K. Chang, "Existence and uniqueness of solutions for third-order nonlinear boundary value problems," Journal of Mathematical Analysis and Applications, vol. 327, no. 1, pp. 23-35, 2007.

[18] Y. Shi and M. Pei, "Two-point and three-point boundary value problems for nth-order nonlinear differential equations," Applicable Analysis, vol. 85, no. 12, pp. 1421-1432, 2006.

[19] L. Wang and M. Pei, "Existence and uniqueness for nonlinear third-order two-point boundary value problems," Dynamics of Continuous, Discrete E Impulsive Systems. Series A, vol. 14, no. 3, pp. 321-332, 2007.

[20] F.-H. Wong, "An application of Schauder's fixed point theorem with respect to higher order BVPs," Proceedings of the American Mathematical Society, vol. 126, no. 8, pp. 2389-2397, 1998.

[21] Q. Yao and Y. Feng, "The existence of solution for a third-order two-point boundary value problem," Applied Mathematics Letters, vol. 15, no. 2, pp. 227-232, 2002.

[22] W. L. Zhao, "Existence and uniqueness of solutions for third order nonlinear boundary value problems," The Tohoku Mathematical Journal, vol. 44, no. 4, pp. 545-555, 1992. 